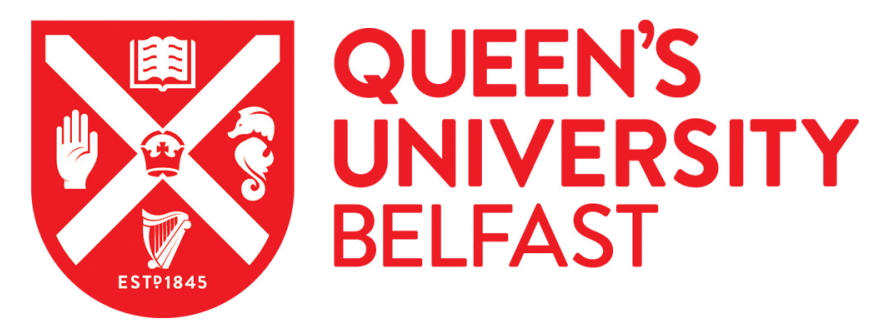

\title{
Evidence for communicative compensation in debt advice with reduced multimodality
}

Andelic, N., Feeney, A., \& McKeown, G. (2019). Evidence for communicative compensation in debt advice with reduced multimodality. In ICMI '19 2019 International Conference on Multimodal Interaction (pp. 210-219). Association for Computing Machinery. https://doi.org/10.1145/3340555.3353757

Published in:

ICMI '19 2019 International Conference on Multimodal Interaction

Document Version:

Peer reviewed version

Queen's University Belfast - Research Portal:

Link to publication record in Queen's University Belfast Research Portal

Publisher rights

Copyright 2019 ACM. This work is made available online in accordance with the publisher's policies. Please refer to any applicable terms of use of the publisher.

\section{General rights}

Copyright for the publications made accessible via the Queen's University Belfast Research Portal is retained by the author(s) and / or other copyright owners and it is a condition of accessing these publications that users recognise and abide by the legal requirements associated with these rights.

Take down policy

The Research Portal is Queen's institutional repository that provides access to Queen's research output. Every effort has been made to ensure that content in the Research Portal does not infringe any person's rights, or applicable UK laws. If you discover content in the Research Portal that you believe breaches copyright or violates any law, please contact openaccess@qub.ac.uk. 


\title{
Evidence for Communicative Compensation in Debt Advice with Reduced Multimodality
}

Nicole Andelic ${ }^{\dagger}$

School of Psychology, Queen’s University Belfast, Belfast, United Kingdom, n.andelic@qub.ac.uk

Aidan Feeney

School of Psychology, Queen’s University Belfast, Belfast, United Kingdom, a.feeney@qub.ac.uk

Gary McKeown

School of Psychology, Queen’s University Belfast, Belfast, United Kingdom, g.mckeown@qub.ac.uk

$\dagger$ Corresponding author.

\begin{abstract}
Research has found that professional advice with empathy displays and signs of listening lead to more successful outcomes. These skills are typically displayed through visual nonverbal signals, whereas reduced multimodal contexts have to use other strategies to compensate for the lack of visual nonverbal information. Debt advice is often a highly emotional scenario but to date there has been no research comparing fully multimodal with reduced multimodal debt advice. The aim of the current study was to compare explicit emotional content (as expressed verbally) and implicit emotional content (as expressed through paralinguistic cues) in face to face (FTF) and telephone debt advice recordings. Twenty-two debt advice recordings were coded as emotional or functional and processed through emotion recognition software. The analysis found that FTF recordings included more explicit emotion than telephone recordings did. However, linear mixed effects modelling found substantially higher levels of arousal and slightly lower levels of valence in telephone advice. Interaction analyses found that emotional speech in FTF advice was characterised by lower levels of arousal than during functional speech, whereas emotional speech in telephone advice had higher levels of arousal than in functional speech. We can conclude that there are differences in emotional content when comparing full and reduced multimodal debt advice. Furthermore, as telephone advice cannot avail of visual nonverbal signals, it seems to compensate by using nonverbal cues present in the voice.
\end{abstract}

\section{CCS CONCEPTS}

- Applied computing Psychology

\section{KEYWORDS}

Affective computing, advice-giving, arousal, valence

\section{Introduction}

People in need of advice, whether it is related to a medical, relational or financial issue, can typically choose to access these services in a variety of ways; in person, over the telephone, and sometimes online. These methods of communication provide contrasting sets of communicative modalities. However, regardless of the mode of communication, there is a need for such advice to be delivered in an understanding and empathic way, especially so when the advice concerns a sensitive matter for the advice recipient. Accordingly, there is a literature devoted to the study of advice practices which lead to the best outcome.

\subsection{Good communication}

The literatures on communication in medical, counselling and sales contexts help us understand the type of communication that lead to positive outcomes. For example, doctor-patient communication is often categorized as "psycho-social" (demonstrating the doctor's soft skills), or "task-oriented" (demonstrating the doctor's informationgiving skills). A meta-analysis of doctor communication and patient adherence [1] has found that psychosocial skills such as empathy, trust-building and patient-centred behaviour lead to improved medication adherence when compared to task-oriented skills, and occasionally psychosocial skills even lead to better health outcomes [2]]. Psychosocial skills are not only associated with adherence and better health: Interaction with patients that include displays of warmth and listening are associated with greater patient satisfaction [3]. 
Although some of these psychosocial skills are carried out verbally, some are displayed through nonverbal behaviour [3]. Accordingly, research has also found that specific nonverbal behaviours are associated with positive outcomes. Doctor displays of nonverbal behaviours, including head nodding and mutual gaze, are associated with better health outcomes [4] and there is evidence of an association between patient satisfaction and doctors' ability to understand nonverbal communication [ $[5]$. The positive influence of nonverbal behaviour has been found in other contexts as well: Nonverbal features of relationship-building, such as non-distancing body language and mimicry, help to ensure positively evaluated service-encounters [6].

What is the mechanism underlying the relationship between nonverbal cues and positive outcomes in communication? Nonverbal cues help the decoder to accurately make judgements about the other person's affective states [7], facilitating explicit emotion work in conversation. One way that visual nonverbal cues may have an advantage in communication is the "mind-reading" hypothesis [ㅇ]. It has been argued that for communication to be successful, the speakers need to take multiple streams of information into account, including visual nonverbal cues and the context of the communication [9]. This allows the speakers to display mind-reading abilities and adjust their communication appropriately. As a consequence, communication that does not have the benefit of full multimodality, such as advice that occurs over the phone, will therefore include less context and visual nonverbal information. Instead, telephone advice is likely to use other, more implicit streams of multimodal communication, such as paralinguistic cues, to compensate for the absence of visual nonverbal signals [10].

From this brief overview we can conclude that there are advantages of advice conversations in which the advisor and advisee engage in emotional work (such as displaying empathy skills). We can also conclude that although reduced multimodal contexts will use compensatory strategies, psychosocial skills may be more easily demonstrated in full multimodal contexts such as face to face advice, as the presence of visual nonverbal signals allow for additional channels of emotion information.

\subsection{Debt advice}

Debt advice is a particularly high-stakes advice scenario as problem debt has associations with stigma [11] and psychological stress [12]. Consequently, debt advice conversations can be highly emotional and previous research on telephone debt advice conversations has found that they are effortful for both the advisor and the advisee [13]. To our knowledge, nobody has compared emotional content in telephone and face to face debt advice.

Although previous studies of the effectiveness of face to face and telephone debt advice produced mixed findings [14] [15], much of that previous research suffers from issues of participant attrition. In addition, debt advice can range from informal budgeting tips to advice on insolvency mechanisms such as bankruptcy [투] . A rare study examining the effectiveness of face to face and telephone advice appointments regarding formal debt resolution mechanisms [17], found that face to face clients were more likely to get their insolvency arrangement approved than clients who communicated using the telephone only. For debt advice then, there seems to be an advantage to advice delivered using multimodal communication, but it is not clear why that is.

\subsection{The current study}

In the current study we decided to compare the emotional content in voice recordings made during full multimodal (face to face) and reduced multimodality (telephone only) debt advice appointments. By collaborating with a private company offering formal debt resolution advice, we could be certain that large portions of the advice appointment will be standardised across both face to face and telephone advice modes. In line with the distinctions made in the literature on doctor-patient communication between "task-oriented" and "psycho-social" aspects of advice conversations, we distinguished between functional and other aspects of the verbal conversations. In addition, we analysed the auditory nonverbal characteristics of the conversations, using automatised techniques from the field of emotion recognition in affective computing [18]. This allowed us to recognise emotions on a continuum of Arousal/Valence (also known as the Activation/Valence model [19]).

Although emotions have traditionally been classified as discrete categories [20], the dimensional approach to emotions has the advantage of allowing for nuances of emotions. It also avoids theoretical issues with the discrete approach, such as deciding which emotions to use, or how to differentiate between subtly different emotions. Arousal has been correctly classified in many emotion recognition studies [21], possibly due to the concept of arousal/activation involving the sympathetic nervous system (e.g. increased heart rate variability and higher blood pressure) [22]. This corresponds with speech which becomes louder, faster and higher pitched. How to extract the dimension valence from speech is more controversial due to the difficulty inherent in automatically separating emotions that are close on the valence continuum [21] [18] [23]. Yet there is no doubt that valence is an important facet of emotion. For example, both joyful and angry speech are high in activation, yet most people would be able to distinguish between the two emotions in speech. 
Prior to the study we formed several hypotheses: 1) Due to the large amounts of disclosure and the negative nature of debt advice conversations we expected high levels of implicit and explicit emotional content in all conversations; 2) Due to the absence of visual nonverbal cues to empathy, such as facial expressions, in telephone advice conversations, we predicted that there would be more auditory evidence of emotion in telephone speech in comparison to face to face speech; and 3) Finally, given the facial and nonverbal cues present in face to face speechbased on the research suggesting a link between such cues and outcomes such as communication and empathy-we predicted that there would be a difference in emotional and functional utterances between advice modes. More specifically, as face to face speech allows for additional nonverbal cues, we hypothesised that there would be more utterances classified as emotional rather than functional in face to face speech than telephone speech.

\section{Methods}

\subsection{Design}

The aim of this study was to examine the difference in emotional content between face to face and telephone debt advice. Emotional content was examined through two very different analyses. The first analysis used software to determine the auditory characteristics of the voice. The same software then identified levels of arousal and valence in the voice based on these auditory characteristics. The second analysis assessed human rated annotations which categorised each speaker utterance into emotional or functional speech.

Based on previous research on debt advice outcomes [17] we hypothesised that there would be a difference between the two advice modes. Therefore our study compared the levels of emotion between clients who had received face to face advice with clients who had received advice via telephone. In our auditory characteristics analysis, our dependent variables were Arousal and Valence. Our independent variables were Advice type (Face to face, Telephone), Role (Advisor, Client), Turn (the order of when the speech unit appears in conversation), Type of utterance (Emotional, Functional) and Topic continuation (Initiation, Continuation). We also included Subject and Recording to control for potential individual or environmental differences in the speech. In our second, explicit emotion analysis, we treated each recording as a separate subject. Our dependent variable was proportion of speech categorised as Emotional or Functional, and our between-subjects variable was Advice type.

\subsection{Data}

We used 22 audio recordings of debt advice appointments in our study, 12 of which belonged to the telephone group and ten face to face recordings. Including the seven advisors who participated in the recordings we had 29 speakers in total. The group of ten face to face participants was recruited by the advisors prior to the advice appointment so that the meeting could be recorded. This is dissimilar to the recruitment process for the telephone group in which the advice conversation had been recorded for company training purposes and the primary researcher contacted advisees after their appointments to ask for their consent in using the recordings. The advisors were given specific instructions on how to approach their clients for study participation as well as information sheets and consent forms. This study was approved by the Psychology Research Ethics Committee at Queen's University Belfast.

\subsection{Software}

The face to face appointments were recorded with a Sony ICDPX333 digital voice recorder, while the recordings of telephone appointments were stored and accessed by the researchers through internal company software for training and monitoring purposes. ELAN Annotation Software [24] was used to transcribe and categorise each utterance as emotional or functional speech. Each utterance was extracted into individual .wav sound files through the phonetics software Praat [25]. These sound files were then analysed in the open-source software OpenSMILE [26] using the database OpenEAR [27]. This allowed us to determine the arousal and valence levels of each utterance according to the OpenEAR parameters. The Arousal-Valence component of OpenEar was originally based on the naturalistic SAL and VAM database of emotions [28] and is one of the most commonly used emotion recognition tools [18] [29]. As only one channel was used to record both speakers during the appointments, on average $20 \%$ of each recording could not be used due to overlapping speech.

\subsection{Data processing}

Each recording underwent an extensive amount of preparation in the previously named applications before being ready for analysis. Firstly, each recording was divided into smaller blocks of speech (from the moment a speaker began speaking until the stream of speech ended or the speaker was interrupted by the next speaker) in ELAN 
Annotation software. Each of these slots of speech was then transcribed and categorised as being advisor or client speech, face to face or telephone speech, and emotional or functional speech.

Approximately half of the recordings were also classified as emotional/functional by a second human rater and only the blocks where consensus was reached were used, meaning that the blocks where there was disagreement were discarded. In total, $97.96 \%$ of the twice-annotated blocks were agreed upon, demonstrating a high inter-rater agreement. In all of our telephone recordings the first few turns of conversation were a typical greeting procedure (hello, introduction and 'how are you'). These were removed to reflect the face to face conversations in which the initial greeting had taken place prior to asking for permission to record. For the same reason we also removed the very last turns in the conversation, consisting of conversation closings. Similar to the method used in the doctorpatient literature, speech which involved the advisor or client orienting to emotional features of the conversation, such as empathy displays or story-telling, was categorised as emotional speech. In comparison, speech that was directly related to, or in response to, the advisor script was categorised as functional speech.

After coding the speech units as emotional or non-emotional (functional) speech, we proceeded to also code whether the speech unit was an initiation to a new topic or whether it was a continuation of the previous topic. This was partly because a switch in topic is often characterised by a change in prosodic cues [30] [31], and these auditory characteristics may be identified as having high levels of arousal. If this is the case, then not controlling for them will make it difficult when comparing arousal/valence in emotional and functional speech, as new topics will be introduced with a renewed enthusiasm regardless of whether it is an emotional or functional speech unit. Topic switches were therefore coded as "initiation" and turns which continued the previous topic was coded as "continuous".

Units containing laughter were then removed. This decision was taken due to the ambiguity of laughter in an emotional context [32], as laughter is not always an affiliative mechanism. When this was done, each recording was put through Praat which extracted the annotated slots into individual sound files by using a script built for the purpose. This resulted in a large dataset of units of utterances. Finally, each sound file was analysed using the OpenEAR acoustic features extraction tool which classified emotions according to the parameters set by this tool (energy, F0, MFCC etc.), which are not available outside the team that created OpenEAR. This gave us the arousal and valence levels of each of the sound units. Arousal ranged from $0-1$ whilst valence ranged from -1 to 1 . Although the OpenEAR tool has a feature which automatically labelled each turn as a discrete emotion, we decided not to use it due to the difficulties associated with discrete emotion theory as mentioned in our introduction [33].

The units that were discarded at various stages of our analysis (e.g. greeting, closing, laughter, overlapping speech or neither functional nor emotional speech) were not included in the main Arousal/Valence recognition analysis. However, when calculating the proportion of emotional and functional talk they were included. This was so that we were able to conduct a mixed ANOVA based on the emotional and functional proportion levels. In total, 494 and 988 units of speech were removed in face to face and telephone conversations, respectively.

\subsection{Materials}

The data was analysed using a linear mixed effects model to examine the effect of advice mode on Arousal/Valence.

\section{Results}

\subsection{Arousal and Valence in debt advice}

To get a sense of what the data looked like it is useful to examine the descriptive statistics. The average length of each telephone recording was 36 minutes, whilst face to face recordings lasted on average 60 minutes. The first stage of the analysis preparation was to define each utterance as a separate audio unit, totalling 11797 units of speech. Each unit was categorised as an advisor (6793 units) or client (5004 units) speaking, and whether the recording took place face to face (6640 units) or via telephone (5157 units).

The first step in the analysis was to compare the average level of arousal and valence between the advice modes. In this model, a rising level of Arousal indicated an increase in activation as opposed to low arousal speech which reflects deactivation [34]. A rising level of Valence meant that the speech was becoming more positive in nature, rather than negative.

- T-tests for both variables found significant differences in levels of Arousal, $\mathrm{t}(6106.9)=-50.447, \mathrm{p}<.001$, and Valence, $\mathrm{t}(11118)=-21.403, \mathrm{p}<.001$, when comparing face to face and telephone advice conversations.

- The average level of Arousal was $.06(\mathrm{SD}=.36)$ for face to face speakers, in comparison to $.84(\mathrm{SD}=1.06)$ for telephone speakers. 
We can conclude that there is a large effect size of Advice Type on Arousal $(d=.99)$ [35]. Telephone speakers also had slightly higher levels of Valence, $.05(\mathrm{SD}=.27)$, in comparison to face to face speakers, $-.06(\mathrm{SD}=.27)$, resulting in a small to medium effect size $(\mathrm{d}=.41)$ [35].

\subsection{Explicit emotion}

The next step of the analysis was to compare the level of emotion as coded by the researchers. Each unit of speech was categorised as Emotional or Functional. A higher proportion of the speech in face to face conversations was coded as Emotional than in telephone advice. Overall, 19\% of the speech was categorised as Emotional and $81 \%$ was categorised as Functional. Advisors were less likely to engage in emotional speech (10.4\%) in comparison to clients $(30.6 \%)$.

To find out whether the proportion categorised as Emotional and Functional differed between face to face and telephone advice, a mixed ANOVA was performed. Each recording was treated as a subject, with Advice Type as the between-subjects variable. The proportion of Emotional and Functional talk (as calculated when also including the removed units as "Other") was the within-subjects variable.

- This ANOVA found a significant interaction between Type of utterance and Advice Type, $F(1,20)=4.45$, $p$ $=.048$.

- Telephone participants showed a lower proportion of emotional utterances $(\mathrm{M}=.11, \mathrm{SD}=.21)$ than face to face participants $(\mathrm{M}=.23, \mathrm{SD}=.02)$.

- In contrast, face to face participants had a lower proportion of functional talk $(\mathrm{M}=.75, \mathrm{SD}=.05)$ than telephone participants $(\mathrm{M}=.80, \mathrm{SD}=.04)$.

\subsection{Arousal and Valence from acoustic features}

The aim of the study was to examine the extent to which Arousal and Valence in debt advice conversations was influenced by Advice type, as well as Role, Type of speech (Emotional/Functional speech and Initiation/Continuous). To do this we applied a linear mixed effects model [36] to the data including Advice type, Role, Type of speech and Initiation type as fixed effects. Recording, Speaker (subject), Turn (split into tenths for each advice appointment to reflect the temporal sequence of the appointment) and Arousal/Valence (depending on which variable that was being fitted at the time) were included as random effects. The model was fitted using the function "Imer" from the Rpackage "Ime4" [37] and the fit of the models was then tested using the recommended restricted maximum likelihood and likelihood ratio tests as a way to compare the best model fit [38]. The random effects were included in all models to control for possible effects on Arousal/Valence stemming from these variables rather than the fixed effects. For example, because the measures of Arousal and Valence are based on auditory characteristics, such as pitch and energy, they are likely to vary between individuals and each advice appointment.

\subsubsection{Arousal in speech}

The first step was to run the full model to predict Arousal. The full linear mixed model was significantly different from the null model $\left(\chi^{2}=93536, \mathrm{df}=7, \mathrm{p}<.001\right)$, and demonstrated that an increase in arousal was positively associated with telephone advice (as opposed to face to face; estimated change in Arousal, $\beta=.50, \mathrm{~S} . \mathrm{E}= \pm .12, \mathrm{~F}=$ $18.08, \mathrm{p}<.001)$, as well as negatively associated with Functional speech $(\beta=-.02 \pm .00, \mathrm{~F}=34.80, \mathrm{p}<.001)$ and Continuous speech $(\beta=-.02 \pm 01, \mathrm{~F}=11.85, \mathrm{p}<.001)$. An increase in Arousal was not found to have a significant association with the role Client $(\beta=.02 \pm .10, \mathrm{~F}=.10, \mathrm{p}=.799)$. To find the best fitting model each parameter was excluded one by one until a model was found that explained sufficient amounts of the data without being needlessly complex [39]. The best model was assessed for goodness of fit using the log-likelihood ratio test. An alternative way of measuring goodness of fit is using the AIC or BIC value, and although the current study opted for the loglikelihood ratio test, using $\mathrm{AIC} / \mathrm{BIC}$ values leads to the same results.

Table 1 Linear mixed effects model for Arousal and Valence

\begin{tabular}{|l|l|l|}
\hline Parameters & Arousal model & Valence model \\
\hline Fixed effects & & \\
\hline Intercept & $.29(.09)$ & $-.02(.02)$ \\
\hline Advice type (telephone) & $.51(.12)$ & $-.05(.02)$ \\
\hline Labour type (functional) & $-.02(<.01)$ & $.00(<.01)$ \\
\hline Topic switch (continuous) & $-.02(<.01)$ & $-.00(<.01)$ \\
\hline Random effects & & \\
\hline
\end{tabular}




\begin{tabular}{|l|l|l|}
\hline Parameters & Arousal model & Valence model \\
\hline Speaker & $.05(.22)$ & $.00(.04)$ \\
\hline Recording & $.04(.20)$ & $.00(.03)$ \\
\hline Turn & $.14(.38)$ & $.01(.08)$ \\
\hline Valence & $.92(.96)$ & - \\
\hline Arousal & - & $.21(.46)$ \\
\hline
\end{tabular}

Note: Fixed effects display estimates and SE in brackets, random effects display variance and SD in brackets

Table 1 shows the models that were systematically tested by removing parameters which had little influence on arousal. Through this method it was found that Model 2 was the best fitting model (Log-likelihood $=77343)$. This model included all the random and fixed effects, excluding Role which was the weakest fit and correlated highly with the intercept $(\mathrm{r}=-.51)$. It provided a significantly better fit than Model 3 which had one parameter less $\left(\chi^{2}=11.92, \mathrm{df}\right.$ $=2, \mathrm{p}=.003)$ but was not significantly different from the full model $\left(\chi^{2}=.06, \mathrm{df}=1, \mathrm{p}=.800\right)$. Using this model, it is possible to see that Advice type influenced Arousal, raising it by $.51( \pm .12, \mathrm{~F}=19.30, \mathrm{p}<.001)$ when switching from face to face to telephone advice. However, switching from emotional to functional speech $(\beta=-.02 \pm .00, \mathrm{~F}=34.83, \mathrm{p}$ $<.001)$ and initiation to continuous speech $(\beta=-.02 \pm .01, \mathrm{~F}=11.86, \mathrm{p}<.001)$ were both associated with small decreases in arousal.

\subsubsection{Valence in speech}

In the full model created to predict levels of valence in speech, the variables were the same as in the Arousal model. The only change was made to the random effect of Arousal which replaced Valence in the previous model as the dependent variables had been switched as well to account for changes on the other emotional dimension. Again, as in the Arousal model, the full Valence model was significantly different from the null model $\left(\chi^{2}=137916, \mathrm{df}=7, \mathrm{p}\right.$ $<.001)$. In this model, an increase in Valence was positively associated with a small increase in Functional speech $(\beta$ $=.00 \pm .00, \mathrm{~F}=14.19, \mathrm{p}<.001)$ but negatively associated with advice over telephone $(\beta=-.05 \pm .02, \mathrm{~F}=7.65, \mathrm{p}=.006)$, Client speech $(\beta=-.02 \pm .02, \mathrm{~F}=.74, \mathrm{p}=.404)$ and Continuous speech $(\beta=-.00 \pm .00, \mathrm{~F}=4.49, \mathrm{p}=.034)$. The decrease in Valence when switching from face to face to telephone advice is particularly surprising, considering that the $t$-test found the opposite previously. However, as can be seen, the majority of these effects were marginal and so the weakest parameters were eliminated in order to find a better model fit.

Table 1 shows the best fitting model for predicting valence after removing parameters. This method showed that, again, Model 2 which mimicked the full model excluding Role, was the best fitting model (Log-likelihood $=63$ 167). This model was significantly better than Model $3\left(\chi^{2}=4.46, \mathrm{df}=1, \mathrm{p}=.035\right)$ but not significantly different from the full model $\left(\chi^{2}=.69, \mathrm{df}=1, \mathrm{p}=.406\right)$, and Role was highly correlated with the intercept variable $(\mathrm{r}=-.63)$ which suggests that the removal of the variable Role was justified.

In Model 2, an increase in Valence was weakly associated with Functional speech $(\beta=.00 \pm .00, \mathrm{~F}=12.27, \mathrm{p}<$ $.001)$, but negatively associated with advice over the phone $(\beta=-.05 \pm .02, \mathrm{~F}=7.51, \mathrm{p}=.006)$ and Continuous speech $(\beta=-.00 \pm .00, \mathrm{~F}=4.46, \mathrm{p}=0.35)$. However, the reader is advised to interpret these findings with caution. Due to the large amount of data points, the probability of the findings is less important than the effect size. As the significant predictors for the Valence model (Advice type and Type of speech) both had marginal effect sizes, the findings suggest that the model is in fact evidence for there being little difference in valence between face to face and telephone speech.

\subsubsection{Interactions}

The linear mixed effects model found a significant 2 (Advice type: Face to face, Telephone) x 2 (Type of utterance: Emotional, Functional) interaction predicting Arousal $(\beta=-13 \pm .03, \mathrm{~F}=21.35, \mathrm{p}<.001)$ (see Figure 1).

- In telephone conversations Arousal is higher in Emotional speech $(M=.99, S D=1.19)$ than in Functional $\operatorname{speech}(\mathrm{M}=.81, \mathrm{SD}=1.03)$.

- In contrast, Arousal is higher during Functional speech $(\mathrm{M}=.08, \mathrm{SD}=.33)$ than during Emotional speech $(\mathrm{M}=.02, \mathrm{SD}=.44)$ in face to face advice. 


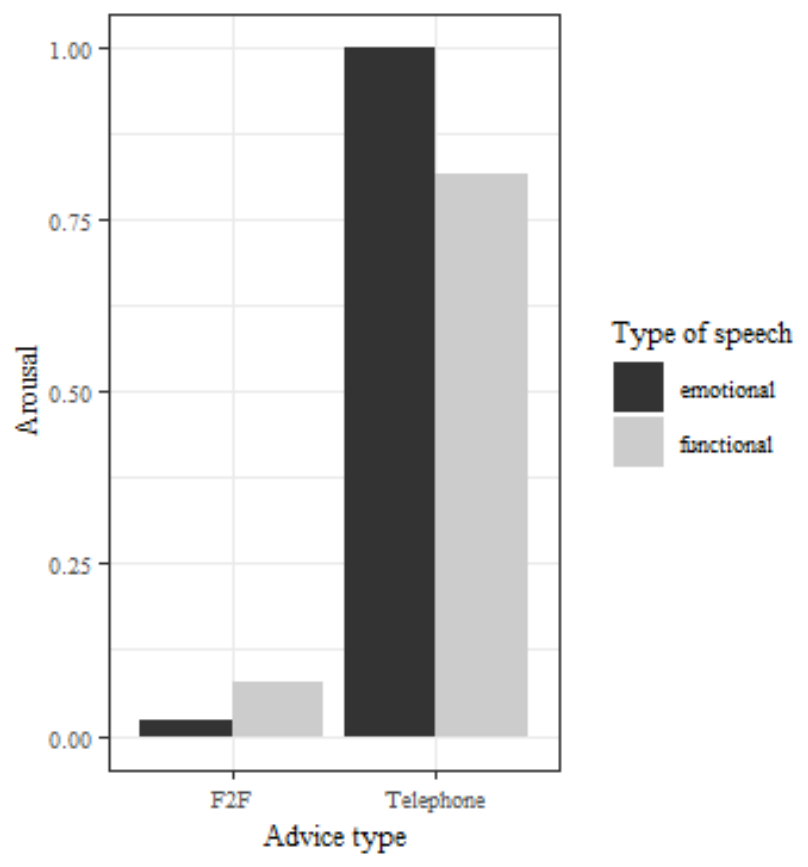

Figure 1 Mean Arousal for Advice type and Type of speech

The second finding was a weak, but significant interaction between Advice type (Face to face, Telephone) and Turn $(\beta=.00 \pm .00, \mathrm{~F}=4.98, \mathrm{p}=.026)$.

- There are only slightly lower levels of Arousal at the start of the face to face conversation than at the end of the conversation.

- In contrast, the pattern of Arousal in telephone conversations manifests itself as a dip just before the middle of the conversation.

This suggests that the pattern of implicit cues to emotion differ between face to face and telephone advice conversations.

\subsubsection{Patterns of explicit and implicit emotion}

In order to see if the pattern of implicit emotion mirrored the explicit emotional content of the conversations we examined the levels of functional and emotional utterances of the conversations, broken down by turn. Although there are no significant increases or decreases in emotional content at the different stages of the face to face conversation (see Figure 2), we are able to see a clear pattern in telephone speech: there is dip in emotional utterances roughly a third into the conversation (see Figure 3, with Arousal in telephone speech displayed as an overlay). When comparing the pattern of implicit emotion (Arousal) of telephone conversations with the pattern of explicit emotion in telephone conversations, we can see that both measures of emotion display this dip. 


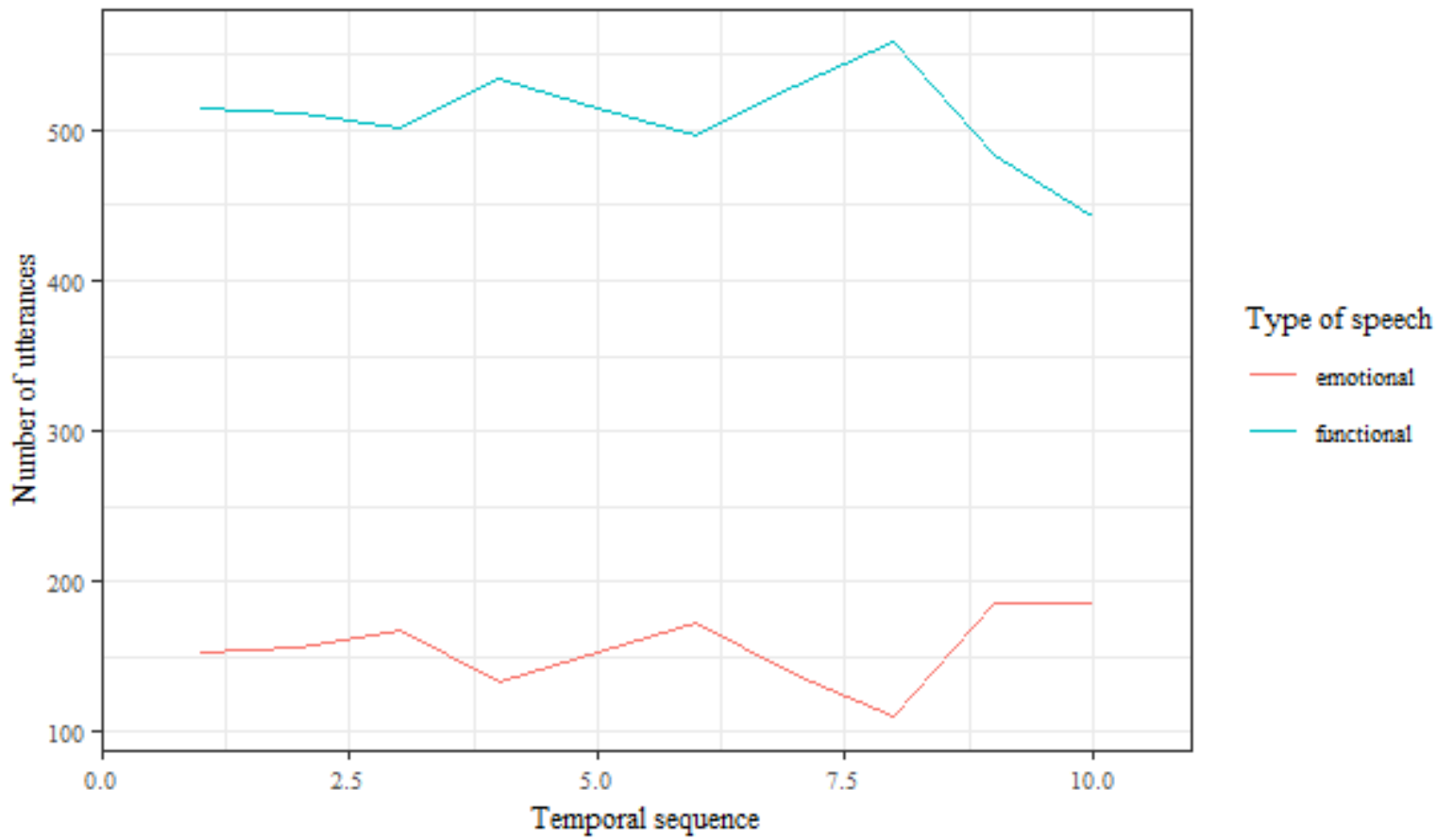

Figure 2 Temporal sequence and Type of speech in Face to face advice

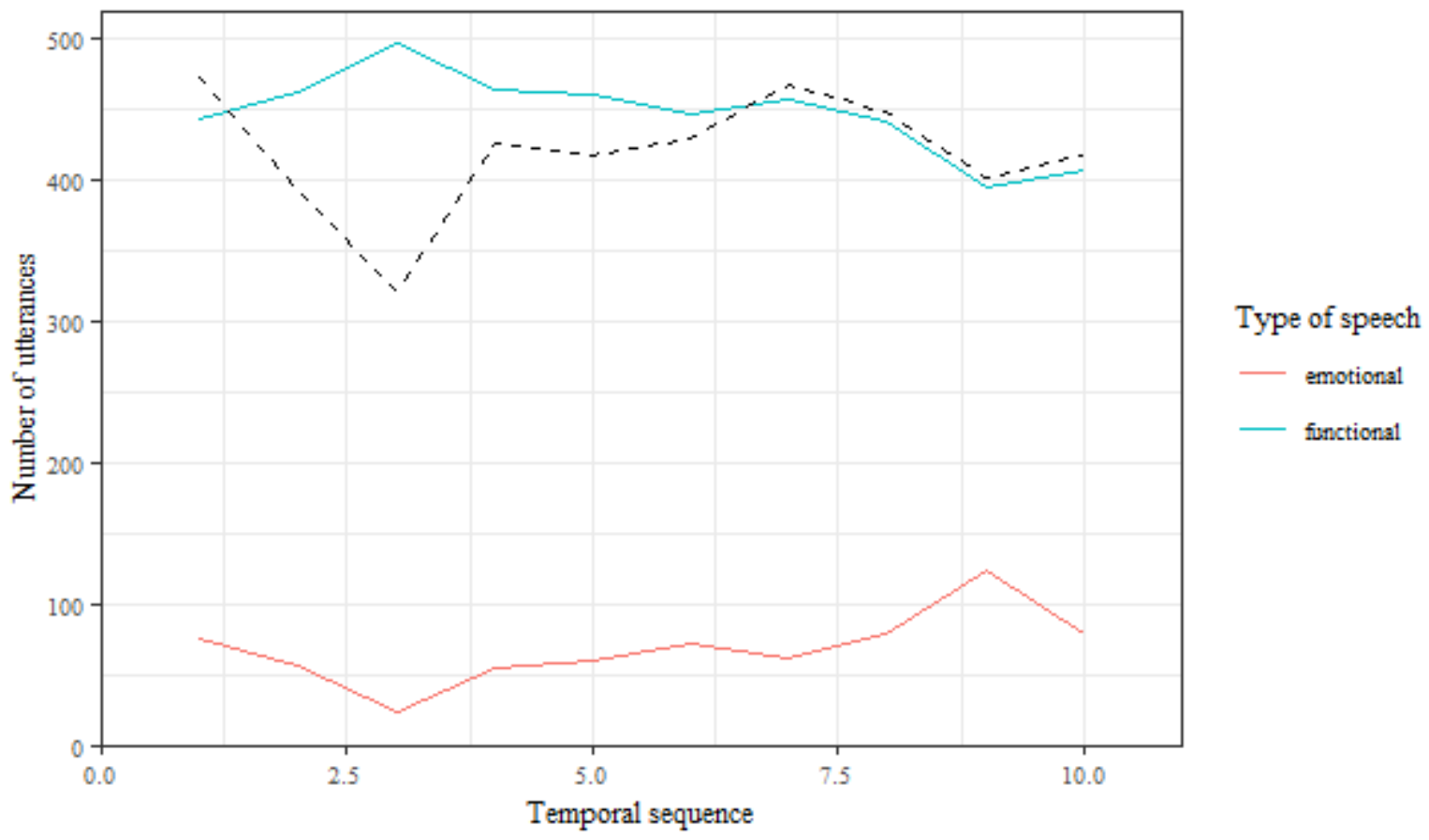

Figure 3 Temporal sequence and Type of speech in Telephone advice. Note that Arousal in telephone speech is displayed as an overlay

\subsubsection{Summary}

To summarise, for both our valence and arousal models we could see that all fixed effects apart from Role had more variability between the factors than within them. The lack of effect when including Role tells us that Valence and Arousal levels do not vary substantially between the advisors and clients. Additionally, Advice type and Emotional/Functional coding have different effects for Arousal and Valence. Our linear mixed models found that 
switching from Face to face to Telephone speech led to an increase in Arousal but a slight decrease in Valence. Switching from Emotional to Functional speech also led to a decrease in Arousal but a very slight increase in Valence, allowing us to conclude that the emotional speech is of a more negative and active nature than the functional portion of the advice conversation. For both Arousal and Valence, we could see that switching from Initiating speech to Continuous speech led to a decrease in both Arousal and Valence. Initiating speech is therefore more active and more positive than Continuous speech.

The interactive effects of Advice type and Turn on Arousal show us that implicit and explicit cues of emotion do not necessarily operate in parallel. For example, although explicit cues to emotional content are higher in face to face conversations than in telephone conversations, implicit paralinguistic cues are higher in telephone conversations than in face to face conversations. The implications of these findings are discussed in the next section.

\section{Discussion}

Our study had three hypotheses: 1) We expected high levels of both explicit and implicit emotional content in both face to face and telephone advice. Our results support this hypothesis, although the levels of arousal differed depending on the advice type. 2) We expected telephone advice to show higher levels of implicit auditory emotion due to the absence of visual cues. We found some support for this hypothesis: telephone advice showed substantially higher levels of arousal in their voice than face to face clients. Additionally, telephone advice also contained lower levels of valence than face to face advice. However, despite the significant result the effect size was small and close to neutral. In practical terms, our results suggest that valence levels are very similar in both advice types. Finally, 3) we predicted that there would be more explicit emotional content in face to face advice than telephone advice. As expected, we found a substantially higher proportion of emotional speech in face to face advice than telephone advice. In addition, although there is no relationship between explicit and implicit emotional cues in face to face advice, when examining telephone advice further, we can see a similar pattern in arousal levels and the proportion of emotional speech. This suggests that telephone advice makes greater use of auditory nonverbal characteristics in the voice to communicate emotion than face to face advice does.

It is possible that when visual nonverbal communication is possible, using the voice to communicate emotion is not necessary. One also says more explicitly emotional things, possibly because the presence of visual nonverbal communication encourages the improvement of the advisor-advisee relationship. In contrast, when there is an absence of visual nonverbal communication, one tends to use the voice to communicate emotion, but despite the use of one's voice there is less explicit emotion being communicated in telephone conversations, possibly due to the lack of nonverbal cues facilitating such disclosure. In summary, there is no correlation between explicit emotion and arousal overall. However, when breaking down the conversations by advice mode, there does seem to be some kind of pattern. For example, in face to face conversations both explicit and implicit emotion remains relatively steady as the conversation unfolds. On the other hand, in telephone conversations emotional utterances dip as arousal dips, and returns to the initial levels at the same time as arousal returns. This suggests that in telephone conversations, participants use their voice to compensate for the lack of visual nonverbal cues. Although participants display less explicit emotion they are using their voice to provide emotional cues when they do.

Overall, these findings support previous research which argues that multimodal encounters allow for more psychosocial communicative gestures than reduced multimodal encounters do [4]. The higher levels of arousal in telephone advice are possibly because using the voice is a way for the speakers to compensate for the absence of visual nonverbal cues. Face to face advice appointments allow the participants to use visual nonverbal communication, which has been shown to increase alignment and empathy through social processes such as mimicry [40] [41]. In contrast, telephone advice participants cannot make use of visual nonverbal cues and they rely on voice only. It is possible that if only voice is available, speakers may utilise auditory characteristics such as pitch variation or voice intensity to emphasise their speech. As a consequence of using these vocal features, arousal levels in the voice would also be higher, which we find in our debt advice conversations. To test this hypothesis, face to face and telephone debt advice appointments should be examined using both voice and visual data. However, due to the sensitive nature of the meetings and commercial restrictions this was not possible when planning the current study.

The larger proportion of explicit emotional content found in face to face advice suggest that there is a larger amount of disclosure of emotional content in these conversations, which could lead to greater rapport-building and trust in the advisor. A decision to apply for a formal debt resolution arrangement is complex and individuals facing such a decision are likely to be under psychological stress, exacerbating the difficulties when making risky decisions [42]. We propose that the ability to use visual nonverbal communication facilitates additional disclosure and troubles-telling opportunities. These lead to greater social alignment with the advisor, which leads to greater engagement with the debt mechanism that is proposed [1]]. 
This study compares emotions in a multimodal and reduced modality setting, but it also adds to the literature on emotion recognition. Automatic emotion recognition can be used in a range of contexts [43], and our findings suggest that there are differences between face to face and telephone contexts that need to be considered when using these tools in an applied setting. Currently, much of the data in emotion recognition research uses acted data rather than natural data [44]. Current research that uses natural data is often confined to data that does not have particularly intense emotions, perhaps because they are reactions to other stimuli (e.g. Wizard-of-Oz scenarios [45]) or is limited in nature due to ethical considerations. Natural data also often has a focus one type of emotion because of the context they are recorded in. For example, recordings in a call center will mainly contain emotions related to anger and have little emotion variability [46] [47] [48]. In contrast, the current study offers relatively long recordings of natural data, which allows us to see the subtle shifts in emotion and emotion intensity. This study also allows us to examine the relationship between explicit emotional content in utterances and the auditory characteristics of those utterances. Although the relationship seems to travel in opposite directions when we compare face to face and telephone conversations, within each type of advice mode implicit and explicit cues show similar patterns: In face to face conversations both are relatively steady, whereas both implicit and explicit cues increase and decrease at similar times in the telephone conversations.

There are some limitations of the current study that should be considered when interpreting our results. Although these findings suggest that speakers engage in more emotional talk in the face to face conversations, it is not possible in the current paradigm to rule out that this could be due to self-selection bias; it is possible that individuals who choose face to face conversations are also more likely to need emotional support than telephone participants. As a consequence, the face to face advisor would engage in more empathetic displays and this could account for the higher levels of emotional content found in face to face advice. Face to face participants were also recruited to the study prior to them having their advice conversations and although both groups were aware of the conversation being recorded, this was made more salient in face to face conversations as the recorder was visible. Although our results support the theory of using the voice as a compensatory strategy, it is possible that there are other reasons for our findings. For example, it is possible that the differences between face to face and telephone are due to social pressure felt in face to face conversations. Future research should explore this theory further by distinguishing between emotional and social labour in speech.

We can conclude that the current study has identified some differences in emotional content between face to face and telephone advice, such as differences in levels of arousal, valence and proportion of speech that consists of emotional talk as opposed to non-emotional talk. The increase in arousal suggests that during telephone advice speakers engage with strategies to compensate for the lack of visual nonverbal streams of information. However, we do not know which of these differences, if any, are the reason why face to face advice leads to higher approval rates. Future research should examine the effectiveness of the compensatory strategies used in scenarios of reduced modality.

\section{ACKNOWLEDGMENTS}

The study was jointly funded by the Economic and Social Research Council [ES/S010882/1], the Business Alliance Office at Queen's University Belfast and Aperture IVA, the company whose clients participated in the research.

\section{REFERENCES}

$<$ bib id="bib1" $><$ number $>[1]</$ number $>$ K. B. Zolnierek and M. H. DiMatteo, "Physician communication and patient adherence to treatment: a metaanalysis," Medical Care, vol. 47, pp. 826-834, 2009.</bib $>$

$<$ bib id="bib2" $><$ number $>[2]</$ number $>$ S. A. Flocke, K. C. Stange and M. A. Goodwin, "Patient and visit characteristics associated with opportunistic preventive services delivery.," Journal of Family Practice, vol. 47, no. 3, pp. 202-208, 1998.</bib>

$<$ bib id="bib3" $><$ number $>[3]</$ number $>$ S. G. Henry, A. Fuhrel-Forbis, M. A. Rogers and S. Eggly, "Association between nonverbal communication during clinical interactions and outcomes: a systematic review and meta-analysis," Patient Education \& Counseling, vol. 86, pp. 297-315, 2012.</bib> $<$ bib id="bib4" $><$ number $>[4]</$ number $>$ R. S. Beck, R. . Daughtridge and P. D. Sloane, "Physician-patient communication in the primary care office: a systematic review.," Journal of The American Board of Family Practice, vol. 15, no. 1, pp. 25-38, 2002.</bib>

$<$ bib id="bib5" $><$ number $>[5]</$ number $>$ M. R. DiMatteo, A. Taranta, H. S. Friedman and L. M. Prince, "Predicting Patient Satisfaction from Physicians' Nonverbal Communication Skills," Medical Care, vol. 18, no. 4, pp. 376-387, 1980.</bib >

$<$ bib id="bib6" $><$ number $>[6]</$ number $>$ D. D. Gremler and K. P. Gwinner, "Rapport-Building Behaviors Used by Retail Employees," Journal of

Retailing, vol. 84, no. 3, pp. 308-324, 2008.</bib>

$<$ bib id="bib7"><number $>[7]</$ number $>$ J. A. Hall, T. G. Horgan and N. A. Murphy, "Nonverbal communication," Annual Review of Psychology, vol. 70, pp. 271-294, 2019.</bib $>$

$<$ bib id="bib8" $><$ number $>[8]</$ number $>$ G. . McKeown, "The Analogical Peacock Hypothesis: The sexual selection of mind-reading and relational cognition in human communication.," Review of General Psychology, vol. 17, no. 3, pp. 267-287, 2013.</bib>

$<$ bib id="bib9"><number $>[9]</$ number $>$ G. . McKeown, "Turing's menagerie: Talking lions, virtual bats, electric sheep and analogical peacocks: Common ground and common interest are necessary components of engagement,", 2015. [Online]. Available:

https://pure.qub.ac.uk/portal/en/publications/turings-menagerie(581da950-ca20-41a1-9432-5f4aad6f557f).html. [Accessed 304 2019].</bib> $<$ bib id="bib10" ><number $>[10]</$ number $>$ J. K. Burgoon, J. A. Bonito, A. R. Jr., N. E. Dunbar, K. . Kam and J. . Fischer, "Testing the interactivity principle: Effects of mediation, propinquity, and verbal and nonverbal modalities in interpersonal interaction," Journal of Communication, vol. 52, no. 3, pp. $657-677,2002 .</$ bib $>$ 
$<$ bib id="bib11"><number $>[11]</$ number $>$ T. . Hayes, "Stigmatizing Indebtedness: Implications for Labeling Theory," Symbolic Interaction, vol. 23, no. 1 , p. $29-46, .</$ bib $>$

$<$ bib id="bib12" $><$ number $>[12]</$ number $>$ T. Richardson, P. Elliott and R. Roberts, "The relationship between personal unsecured debt and mental and physical health: a systematic review and meta-analysis.," Clinical Psychology Review, vol. 33, pp. 1148-1162, 2013.</bib>

$<$ bib id="bib13"><number $>[13]</$ number $>$ N. Andelic, C. Stevenson and A. Feeney, "Managing a moral identity in debt advice conversations," British Journal of Social Psychology, vol. Advance online publication, 2018.</bib $>$

$<$ bib id="bib14" >< number $>[14]</$ number $>$ Money Advice Service, "The effectiveness of debt advice in the UK," YouGov, London, 2010.</bib $>$

$<$ bib id="bib15" $><$ number $>[15]</$ number $>$ Money Advice Service, "Debt advice outcomes framework," Personal Finance Research Centre, Bristol,

2013.</bib>

$<$ bib id="bib16" $><$ number $>[16]</$ number $>$ Money Advice Service, "The economic impact of debt advice," Europe Economics, London, 2018.</bib>

$<$ bib id="bib17" $><$ number $>[17]</$ number $>$ N. Andelic, The Psychology of Debt Advice (Unpublished Dissertation), Belfast: Queen's University Belfast, 2018. $<$ /bib $>$

$<$ bib id="bib18" $><$ number $>[18]<$ number $>$ B. W. Schuller, S. . Steidl, A. . Batliner, F. . Burkhardt, L. . Devillers, C. A. Müller and S. . Narayanan,

"Paralinguistics in speech and language-State-of-the-art and the challenge," Computer Speech \& Language, vol. 27, no. 1, pp. 4-39, 2013.</bib>

$<$ bib id="bib19" $><$ number $>[19]</$ number $>$ R. . Picard, Affective Computing, ed., vol. , , : MIT Press, , p. 1.</bib $>$

$<$ bib id="bib20" $><$ number $>[20]</$ number $>$ K. R. Scherer, "Psychological models of emotion," in The Neuropsychology of Emotion, Oxford/New York, Oxford University Press, 2000, pp. 137-162.</bib $>$

$<$ bib id="bib21" $><$ number $>[21]</$ number $>$ K. R. Scherer, "Vocal communication of emotion: a review of research paradigms," Speech Communication, vol. 40 , no. 1 , pp. $227-256,2003 .</$ bib $>$

$<$ bib id="bib22" $><$ number $>[22]</$ number $>$ M. M. H. E. Ayadi, M. S. Kamel and F. . Karray, "Survey on speech emotion recognition: Features,

classification schemes, and databases," Pattern Recognition, vol. 44, no. 3, pp. 572-587, 2011.</bib>

$<$ bib id="bib23" $><$ number $>[23]</$ number $>$ R. . Tato, R. . Santos, R. . Kompe and J. M. Pardo, "Emotional space improves emotion recognition," , 2002.

[Online]. Available: http://www-gth.die.upm.es/partners/sony/icslp2002.pdf. [Accessed 304 2019].</bib>

$<$ bib id="bib24" $><$ number $>[24]</$ number $>$ H. Brugman and A. Russell, Annotating multimedia: Multi-modal resources with ELAN, 2004.</bib $>$

$<$ bib id="bib25" $><$ number $>[25]</$ number $>$ P. . Boersma, "Praat: doing Phonetics by Computer," , . [Online]. Available:

http://www.fon.hum.uva.nl/praat/. [Accessed 3042019$]$. $<$ /bib $>$

$<$ bib id="bib26" $><$ number $>[26]</$ number $>$ F. Eyben, M. Wöllmer and B. Schuller, "Opensmile: the munich versatile and fast open-source audio feature extractor," in Proceedings of the 18th ACM international conference on Multimedia, Firenze, $2010 .</$ bib $>$

$<$ bib id="bib27" $><$ number $>[27]</$ number $>$ F. Eyben, M. Wöllmer and B. Schuller, "OpenEAR-introducing the Munich open-source emotion and affect recognition toolkit," in 2009 3rd international conference on affective computing and intelligent interaction and workshops, Amsterdam, 2009.</bib> $<$ bib id="bib28" $><$ number $>[28]</$ number $>$ M. Grimm, K. Kroschel and S. Narayanan, "Support vector regression for automatic recognition of spontaneous emotions in speech,” in 2007 IEEE International Conference on Acoustics, Speech and Signal Processing-ICASSP'07, Honolulu, 2007.</bib>

$<$ bib id="bib29" $><$ number $>[29]</$ number $>$ A. Stuhlsatz, C. Meyer, F. Eyben, T. Zielke, G. Meier and B. Schuller, "Deep neural networks for acoustic emotion recognition: Raising the benchmarks," in 2011 IEEE international conference on acoustics, speech and signal processing (ICASSP), Prague, 2011.</bib >

$<$ bib id="bib30" $><$ number $>[30]</$ number $>$ M. Lennes and H. Anttila, "Prosodic features associated with the distribution of turns in finnish informal dialogues," in The Phonetics Symposium, Helsinki, 2002.</bib $>$

$<$ bib id="bib31" $><$ number $>[31]</$ number $>$ G. Tür, D. Hakkani-Tür, A. Stolcke and E. Shriberg, "Integrating prosodic and lexical cues for automatic topic segmentation," Computational linguistics, vol. 27, pp. 31-57, 2001.</bib>

$<$ bib id="bib32" $><$ number $>[32]</$ number $>$ P. . Glenn, "Laughter in Interaction by Phillip Glenn," , 2003. [Online]. Available:

https://cambridge.org/core/books/laughter-in-interaction/4629463a15293cfebd21ee70aac966f2. [Accessed 304 2019].</bib>

$<$ bib id="bib33" $><$ number $>[33]<$ /number $>$ K. R. Scherer, "What are emotions? And how can they be measured?," Social Science Information, vol. 44, pp. 695-729, 2005.</bib $>$

$<$ bib id="bib34" $><$ number $>[34]</$ number $>$ L. F. Barrett, "Discrete Emotions or Dimensions? The Role of Valence Focus and Arousal Focus," Cognition \& Emotion, vol. 12, no. 4, pp. 579-599, 1998.</bib>

$<$ bib id="bib35" $><$ number $>[35]</$ number $>$ J. Cohen, Statistical power analysis for the behavioural sciences, New York: Routledge, 1988. $<$ bib $>$ $<$ bib id="bib36" $><$ number $>[36]</$ number $>$ R. H. Baayen, D. J. Davidson and D. M. Bates, "Mixed-effects modeling with crossed random effects for subjects and items," Journal of Memory \& Language, vol. 59, pp. 390-412, 2008.</bib $>$

$<$ bib id="bib37"><number $>[37]</$ number $>$ D. M. Bates, M. . Mächler, B. M. Bolker and S. C. Walker, "Fitting Linear Mixed-Effects Models Using lme4," Journal of Statistical Software, vol. 67, no. 1, pp. 1-48, 2015.</bib $>$

$<$ bib id="bib38" $><$ number $>[38]</$ number $>$ J. C. Pinheiro and D. M. Bates, "Linear mixed-effects models: basic concepts and examples," Mixed-effects models in S and S-Plus, pp. 3-56, 2000.</bib $>$

<bib id="bib39" $><$ number $>[39]</$ number $>$ J. L. Rodgers, "The epistemology of mathematical and statistical modeling: a quiet methodological revolution," American Psychologist, vol. 65, pp. 1-12, 2010.</bib>

$<$ bib id="bib40" $><$ number $>[40]</$ number $>$ J. L. Lakin and T. L. Chartrand, "Using nonconscious behavioral mimicry to create affiliation and rapport,"

Psychological Science, vol. 14, pp. 334-339, 2003.</bib $>$

$<$ bib id="bib41" $><$ number $>[41]</$ number $>$ L. Menenti, S. C. Garrod and M. J. Pickering, "Toward a neural basis of interactive alignment in conversation," Frontiers in Human Neuroscience, vol. 6, pp. 1-9, 2012.</bib $>$

$<$ bib id="bib42" $><$ number $>[42]</$ number $>$ F. C. Murphy, J. S. Rubinsztein, A. . Michael, R. D. Rogers, T. W. Robbins, E. S. Paykel and B. J. Sahakian,

"Decision-making cognition in mania and depression," Psychological Medicine, vol. 31, no. 4, pp. 679-693, 2001.</bib>

$<$ bib id="bib43"><number $>[43]</$ number $>$ V. Petrushin, "Emotion in speech: Recognition and application to call centers.," in Proceedings of Artificial

Neural Networks In Engineering (ANNIE), 1999.</bib>

$<$ bib id="bib44" ><number $>[44]</$ number $>$ S. Yildirim, M. Bulut, C. M. Lee, A. Kazemzadeh, Z. Deng, S. Lee, S. Narayanan and C. Busso, "An Acoustic Study of Emotions Expressed in Speech," in 8th International Conference on Spoken Language Processing, Jeju Island, 2004.</bib>

$<$ bib id="bib45" $><$ number $>[45]</$ number $>$ A. Batliner, S. Steidl, C. Hacker and E. Nöth, "Private emotions versus social interaction: a data-driven approach towards analysing emotion in speech," User Modeling and User-Adapted Interaction, vol. 18, pp. 175-206, 2008.</bib>

$<$ bib id="bib46" $><$ number $>[46]</$ number $>$ L. Devillers, C. Vaudable and C. Chastagnol, "Real-life emotion-related states detection in call centers: a cross-corpora study," in Eleventh Annual Conference of the International Speech Communication Association, Makuhari, 2010.</bib>

$<$ bib id="bib47" $><$ number $>[47]</$ number $>$ D. Morrison, R. Wang and L. C. De Silva, "Ensemble methods for spoken emotion recognition in callcentres," Speech Communication, vol. 49, pp. 98-112, 2007.</bib $>$

$<$ bib id="bib48" $><$ number $>[48]</$ number $>$ L. Vidrascu and L. Devillers, "Five emotion classes detection in real-world call center data: the use of various types of paralinguistic features," in Proceedings of the International Workshop on Paralinguistic Speech between models and data, 2007.</bib $>$ 
